\title{
A Male-produced Sex Pheromone from the Neotropical Redbanded Stink Bug, Piezodorus guildinii (W.)
}

\author{
Miguel Borges • Jocelyn G. Millar • R. A. Laumann • \\ Maria C. B. Moraes
}

Received: 8 November 2006 / Revised: 4 March 2007 / Accepted: 13 March 2007 /

Published online: 24 April 2007

(C) Springer Science + Business Media, LLC 2007

\begin{abstract}
The male-produced sex pheromone of the neotropical redbanded stink bug, Piezodorus guildinii (W.) (Hemiptera: Pentatomidae), a serious pest of soybean in Central and South America, was identified as the sesquiterpene hydrocarbon $\beta$-sesquiphellandrene by coupled gas chromatography-mass spectrometry and laboratory bioassays. The identity was confirmed by comparison with an authentic standard, and its absolute configuration was determined to be $(7 R)-(+)-\beta$-sesquiphellandrene. The mean rate of release was approximately $40 \mathrm{ng} \mathrm{d}^{-1}$ male $^{-1}$. The compound was found only in extracts from sexually mature males. Laboratory olfactometer bioassays showed that $200 \mathrm{ng}$ of the synthetic compound applied to a filter paper strip was as attractive to female bugs as odors from five 15 -d-old live males, an airborne extract from males, or the fraction of the extract that contained $(7 R)-(+)-\beta$-sesquiphellandrene.
\end{abstract}

Keywords Soybean pest $\cdot$ Sex pheromone $\cdot \beta$-Sesquiphellandrene $\cdot$ Olfactometer bioassay

\section{Introduction}

The neotropical redbanded stink bug, Piezodorus guildinii (W.) (Hemiptera: Pentatomidae), is one of the complex of stink bugs that are serious pests of soybean, Glycine max (L.) Merryl, in Central and South America, especially in Brazil (Panizzi and Rossi 1991). More than four million liters of chemical insecticides are used in Brazil each year to control these pests (Corrêa-Ferreira and Moscardi 1996). The use of semiochemical-based methods for control, such as pheromone-based mass trapping or attract-and-kill, would be a more ecologically benign approach to management of these stink bugs.

M. Borges $(\bowtie) \cdot$ R. A. Laumann • M. C. B. Moraes

EMBRAPA/Recursos Genéticos e Biotecnologia/NCB-Cx., 02372, Brasília,

DF 70849-970, Brazil

e-mail: mborges@cenargen.embrapa.br

J. G. Millar

Department of Entomology, University of California, Riverside, CA 92521, USA 
The first study of semiochemicals in the genus Piezodorus was carried out by Leal et al. (1998). These authors, working with Piezodorus hybneri (Gmelin), found in Thailand, Korea, Taiwan, Japan, and some regions of India and Australia (Shepard et al. 1983; Singh et al. 1989), reported that the male-produced pheromone consisted of three compounds, $\beta$ sesquiphellandrene, $(R)$-15-hexadecanolide, and methyl $(Z)$-8-hexadecenoate, in the ratio 10:4:1. In laboratory bioassays, both sexes were attracted to this blend.

More recently, Borges et al. (1999) reported the preliminary identification of two possible components of the pheromone blend of $P$. guildinii. Confirmation of these components in the $P$. guildinii pheromone blend and identification of any additional components could provide a species-specific attractant for this species. Thus, the objectives of the study reported here were (1) to collect and analyze the volatiles produced by sexually mature, virgin adults of both sexes of $P$. guildinii; (2) to compare the extracts, looking specifically for sex-specific compounds that might be pheromone components; and (3) to identify, synthesize, and test the biological activity of any such sex-specific compounds.

\section{Methods and Materials}

Insects Piezodorus guildinii were obtained from a laboratory colony started from adults collected from soybean fields between 2003 and 2005 near the EMBRAPA Genetic Resources and Biotechnology Laboratory, Brasília, DF, Brazil $\left(15^{\circ} 47^{\prime} \mathrm{S}\right.$ and $\left.47^{\circ} 55^{\prime} \mathrm{W}\right)$. Bugs were reared on raw peanuts (Arachis hypogaea), soybeans (Glycine max), fresh green beans (Phaseolus vulgaris), and water at $26 \pm 1^{\circ} \mathrm{C}$ and $65 \%$ relative humidity under a 14:10 light and dark photoperiod (light 06:00 to 20:00 hours). Insects were reared in 8-1 plastic containers (100-150/container) with the food supply being renewed three times per week. A $15-\mathrm{cm}^{2}$ plastic mesh ( $\left.40 \mathrm{mesh}\right)$ was placed against the inner wall of each container as an oviposition substrate and shelter for the bugs. Egg masses were collected daily and incubated in 9-cm-inner-diameter (ID) plastic petri dishes until they hatched. After the resulting nymphs molted into second instars, they were transferred to plastic containers and reared as above. To prevent interactions between the sexes, males were separated from females after the imaginal molt and cuticular hardening (ca. $24 \mathrm{hr}$ after molting). Sexually mature adults, $\geq 8 \mathrm{~d}$ after the final molt, were used for all bioassays.

Collection of Volatiles Volatiles were collected ( $N=150$ extracts) from groups of 20 male or female $P$. guildinii. The insects were carefully introduced into 1-1 glass containers to minimize emission of defensive secretions (Zhang et al. 2003). A vacuum was used to draw air $(\sim 1$ 1/min) into the container through a bed of 4-12 mesh-activated charcoal (Fisher Scientific, Pittsburgh, PA, USA), and out of the container through two traps $(15 \times 1.5 \mathrm{~cm}$ outer diameter) containing Super Q (200 mg each; Alltech Associates, Inc., Deerfield, IL, USA).

Collection of volatiles commenced $5 \mathrm{~d}$ after the final molt. Insects were fed with fresh green beans (replaced three times a week) and aerated continuously for $15 \mathrm{~d}$, changing the adsorbent traps daily. The traps were eluted with hexane $(4 \times 0.5 \mathrm{ml})$ and the combined eluates stored at $-30^{\circ} \mathrm{C}$ until used for chemical analysis or behavioral bioassay. To check for background volatiles, the volatiles from green beans alone were collected as described above, over four consecutive days $(N=12)$.

Extracts were concentrated under a gentle stream of $\mathrm{N}_{2}$ to yield a solution of approximately 0.1 bug-equivalent $(\mathrm{BE}) / \mu \mathrm{l}$, where $1 \mathrm{BE}$ was the amount produced by one male bug in $1 \mathrm{~d}$. For quantitative analysis, an internal standard [25 $\mu \mathrm{g}(E)$-2-octen-1-ol in $50 \mu \mathrm{l}$ of hexane] was added to the solution. 
To evaluate the circadian rhythm of pheromone release, volatiles were collected and quantified during three different periods: morning, from 07:00 hours to noon $(N=7)$; afternoon, noon to 17:00 hours $(N=5)$; and evening, 17:00 to 22:00 hours $(N=5)$.

Analysis and Fractionation of Extracts Crude extracts and fractions thereof were analyzed on a PerkinElmer gas chromatograph (GC; DB-1 column, $30 \mathrm{~m} \times 0.25 \mathrm{~mm} \mathrm{ID,} 0.25 \mu \mathrm{m}$ film; J\&W Scientific, Folsom, CA, USA) equipped with a flame ionization detector (FID), using a temperature program of $50^{\circ} \mathrm{C}$ (for $2 \mathrm{~min}$ ) increased by $15^{\circ} \mathrm{C} / \mathrm{min}$ to $250^{\circ} \mathrm{C}$. Crude extracts were also analyzed at the University of California, Riverside, with a HewlettPackard 6890 GC interfaced to an HP-5973 mass selective detector, with electron impact ionization $(70 \mathrm{eV})$. A DB-5 MS column was used $(30 \mathrm{~m} \times 0.25 \mathrm{~mm} \mathrm{ID,} 0.25 \mu \mathrm{m}$ film thickness; J\&W Scientific), with a temperature program of $40^{\circ} \mathrm{C}$ (for $1 \mathrm{~min}$ ), $10^{\circ} \mathrm{C} / \mathrm{min}$ to $250^{\circ} \mathrm{C}$, injector $250^{\circ} \mathrm{C}$, and transfer line $280^{\circ} \mathrm{C}$. Injections were made in splitless mode.

An extract was fractionated as described by Leal et al. (1998). A column $(6.0 \mathrm{~cm} \times$ $0.5 \mathrm{~cm}$ ID) containing $500 \mathrm{mg}$ of silica gel (80/100 mesh), impregnated by $10 \%$ with weight $\mathrm{AgNO}_{3}$ (Merck, Darmstadt, Germany), was conditioned by eluting with $6 \mathrm{ml}$ of $25 \%$ ether in hexane, followed by $6 \mathrm{ml}$ of hexane. Three 24-hr aeration extracts from 20 mature males in $\sim 1 \mathrm{ml}$ pentane were loaded onto the column, and eluted sequentially with $3 \times 1 \mathrm{ml}$ pentane, $3 \times 1 \mathrm{ml} 5 \%$ ether in pentane, $3 \times 1 \mathrm{ml} 10 \%$ ether in pentane, and $3 \times 1 \mathrm{ml} 100 \%$ ether, collecting each aliquot as a separate fraction. The first two fractions of $10 \%$ ether in pentane, containing primarily $\beta$-sesquiphellandrene, were combined (fraction A). All the remaining fractions were recombined into one solution (fraction B) for bioassay.

Components in extracts from both sexes of $P$. guildinii were tentatively identified by comparison of mass spectral data with library data (NIST-Wiley database, 2000). Identifications were confirmed by comparison of retention times and mass spectra of insect-produced compounds with those of authentic standards.

Determination of the Absolute Configuration of $\beta$-Sesquiphellandrene The determination of the absolute configuration of the $P$. guildinii male-specific sesquiterpene, $\beta$-sesquiphellandrene, was carried out as described by McBrien et al. (2002) (Fig. 1). The enantiomers of $\beta$ sesquiphellandrene were not resolved on a Cyclodex B chiral stationary phase GC column (J\&W Scientific), but the column did resolve the enantiomers of the related compound, $\alpha$ curcumene. Consequently, an aliquot of extract (containing $\beta$-sesquiphellandrene) was dehydrogenated to $\alpha$-curcumene (Jackman 1960) by treatment with $100 \mu$ l of a benzene solution of 1,2,3,4-tetrachlorobenzoquinone $(20 \mathrm{mg} / \mathrm{ml})$ in a sealed vial at $80^{\circ} \mathrm{C}$ for $1 \mathrm{hr}$. The cooled reaction mixture was filtered through a $1-\mathrm{cm}$ plug of silica gel, eluted with pentane, and the eluate analyzed with a Cyclodex B column (30 $\mathrm{m} \times 0.25 \mathrm{~mm}$ ID) operated isothermally at $90^{\circ} \mathrm{C}(140 \mathrm{kPa}$ helium head pressure). Authentic samples of $(+)-$ and $(-)-\beta$-sesquiphellandrene (see below) were dehydrogenated in identical fashion to provide standards for comparison.

Fig. 1 Enantiomers of sesquiphellandrene. $(7 R)-(+)-\beta-$ Sesquiphellandrene is produced by male P. guildinii

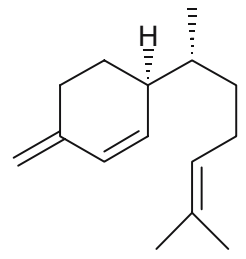

$(7 R)-(+)-\S-s e s q u i p h e l l a n d r e n e$

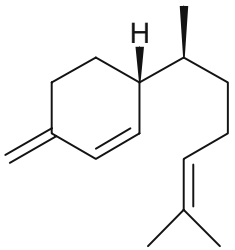

(7S)-(-)-§-sesquiphellandrene 
A second aliquot of the insect-produced $\beta$-sesquiphellandrene was hydrogenated by stirring it with $5 \% \mathrm{Pd}$ on carbon catalyst under $\mathrm{H}_{2}$ for $1 \mathrm{hr}$. This produced two diastereomers in a ratio of $2.5: 1$. Samples of synthetic $\beta$-sesquiphellandrene enantiomers (see below) and a sample of $(S)$ - $\beta$-sesquiphellandrene isolated from ginger oil (McBrien et al. 2002) were hydrogenated in identical fashion to provide standards. All samples were analyzed isothermally $\left(75^{\circ} \mathrm{C}\right)$ on the Cyclodex B column.

Olfactometer Bioassays A two-choice olfactometer modified from Borges and Aldrich (1994) was used to test the biological activity of odors from live insects, aeration extracts of male $P$. guildinii, and fractions thereof. The olfactometer consisted of a 500-ml three-neck, roundbottom flask (24/40 joints, Kontes, Vineland, NJ, USA). Two 250-ml rotary evaporator trap adapters (24/40 joints) were attached to the two outside arms to provide treatment and control chambers. A charcoal filter (20/40 mesh, $130 \times 10-\mathrm{mm}$ ID), connected in series to a water bubbler to humidify the incoming air, was attached to the inlets of the two chambers with silicon rubber tubing (Silastic, $4.8 \mathrm{~mm}$ ID; VWR Scientific, Darmstadt, Germany). Air was pulled through the system $(400 \mathrm{ml} / \mathrm{min})$ by connecting the middle neck of the flask to a regulated vacuum source, with the flow measured with a Clear Flow Rotameter (Accura Flow Products, Warminster, PA, USA). The olfactometer was positioned horizontally on a countertop in a room with fluorescent lighting $(4 \times 40 \mathrm{~W})$. Temperature in the room was maintained at $26.0 \pm$ $1.0^{\circ} \mathrm{C}$. Positions of control and treatment arms were alternated between replicates to avoid any positional bias. The apparatus was cleaned after each five replicates with fragrance-free liquid soap, rinsed thoroughly with water, and dried in a convection oven at $80^{\circ} \mathrm{C}$.

To begin an assay, a single $P$. guildinii was gently introduced into the release chamber with an artist's paint brush (Camel Hair, number 1), and allowed to acclimate for $3 \mathrm{~min}$ while the remainder of the apparatus was assembled. After attaching the treatment and control chambers and starting the airflow, the test bug's behavior was recorded over $15 \mathrm{~min}$. We recorded the first arm of the olfactometer that each insect chose and subsequently remained in for at least $100 \mathrm{sec}$. Insects that did not leave the release chamber of the olfactometer during the first 10 min were considered "nonresponders," and were not included in the data analyses.

To determine which sex produced pheromone, we tested the attraction of insects of both sexes to odor from males $(N=51$ for females and $N=30$ for males) or females $(N=54$ for females and $N=32$ for males). Test individuals were offered a choice between odor from five live, sexually mature virgin insects or a clean air control. The five live insects used as odor sources were changed after 10 replicates.

Bioassays using crude extracts from males were performed with females $(N=74)$ or males $(N=30)$. Five microliters $(0.5 \mathrm{BE})$ of crude hexane extract of the volatiles produced by $20 \mathrm{P}$. guildinii males aerated for $24 \mathrm{hr}$ were spotted on a strip of filter paper, and the paper was placed in one of arm of the olfactometer. Extracts used in these tests contained

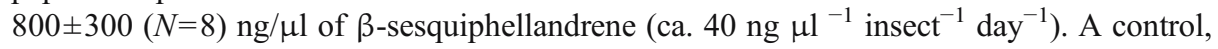
consisting of a filter paper treated with hexane was placed in the other arm.

To determine when females were most active and responsive to pheromone, bioassays were performed during three periods: morning (07:00 hours to noon) $(N=23)$, afternoon (noon to 17:00 hours) $(N=36)$, and evening (17:00 hours to 22:00 hours) $(N=64)$. For these bioassays the methodology described above was followed.

To test the biological activity of crude extracts of males, fractions of extracts, and synthetic standards, and solutions of test stimuli $(1 \mathrm{BE})$ were spotted on a strip of filter paper ( $1.5 \mathrm{~cm}$ long, $0.5 \mathrm{~cm}$ wide), and placed in one of the arms of the bioassay apparatus. Controls, consisting of filter papers treated with hexane, were placed in the other arm. Two 
hundred nanograms of each synthetic standard were used in the bioassays. Bioassays were carried out during the evening period, using virgin, sexually mature females.

Fraction $\mathrm{A}$, containing primarily $\beta$-sesquiphellandrene $(N=70)$, and fraction $\mathrm{B}$, containing the other volatiles released by male $P$. guildinii $(N=34)$, were tested against hexane controls. Fractions $\mathrm{A}$ and $\mathrm{B}$ were also directly compared $(N=30)$, as was fraction A against crude extract $(N=30)$ or $(7 S)-(-)-\beta$-sesquiphellandrene $(N=30)$ isolated from ginger oil. Synthetic $(7 R)-(+)$ and $(7 S)-(-)-\beta$-sesquiphellandrenes were tested against $n$-hexane controls $(N=55$ and $N=54$, respectively). In further tests, females were tested with the following comparisons: fraction A vs. synthetic $(7 R)-(+)-\beta$-sesquiphellandrene $(N=39)$ or synthetic $(7 S)-(-)-\beta$ sesquiphellandrene $(N=40) ;(7 R)-(+)$ vs. $(7 S)-(-)-\beta$-sesquiphellandrene $(N=80)$; and a 1:1 mixture of synthetic $(7 R)-(+)$ and $(7 S)-(-)-\beta$-sesquiphellandrenes vs. each enantiomer individually $[N=72$ for $(R)$ and $N=65$ for $(S)]$. In addition, the biological activities of the last treatments were evaluated in bioassays with males ( $N=27$ and $N=34$, respectively).

Data on the responses of males and females to different treatments (live insects, aeration extracts and fractions thereof, and synthetic standards vs. controls) were analyzed with chisquare tests. Statistical analyses were carried out by using MathCAD 2001 (MathSoft). Data on the production of pheromone during different periods of the day were analyzed with repeated measures ANOVA (Sigma Stat 3.1 Systat Software Inc.).

Chemicals The synthetic sesquiphellandrenes were synthesized from $(R)-(+)$-citronellal and (S)-(-)-citronellal, respectively (Aldrich Chemical, Co., Milwaukee, WI, USA), by the method of Chavan et al. (1997). Each consisted of an inseparable, 1:1 mixture of the resulting sesquiphellandrene enantiomer with its $\mathrm{C} 6$ ring-junction epimer. The enantiomeric purity of the $(7 R)$-isomer was $84 \%$ enantiomeric excess (ee) (reflecting the enantiomeric purity of the citronellal starting material), whereas the $(7 S)$-enantiomer had an optical purity of $>98 \%$. In addition, enantiomerically pure $(7 S)$-sesquiphellandrene was isolated from ginger oil as previously described (Millar 1998). $\beta$-Bisabolene and $\alpha$-curcumene were available from previous studies (McBrien et al. 2002), (E)- $\beta$-farnesene was purchased from Bedoukian Research (Danbury, CT, USA), and nonanal was obtained from Avocado Research (Heysham, UK). Samples of cis- and trans- $\beta$-ocimene were kindly provided by Peter Landolt (US Department of Agriculture, Yakima, WA, USA). All other authentic standards were purchased from Aldrich.

\section{Results}

Analyses of Extracts of Volatiles Comparison of gas chromatograms and mass spectra of volatile compounds collected from sexually mature virgin males and females showed a number of compounds in common (Table 1). Most compounds had been reported previously from other stink bug species (Borges and Aldrich 1992; Zarbin et al. 2000), including $(E)$-2-hexenal, $(E)$ - and $(Z)$ - $\beta$-ocimene, 2-ethylhexanol, $(E)$-2-hexenyl acetate, undecane, nonanal, dodecane, tridecene, and tridecane. In addition, the extracts contained variable amounts of a group of compounds that were tentatively identified as sesquiterpenes, based on their molecular ions $(\mathrm{m} / \mathrm{z} 204$ or 202); the general profiles of their mass spectra, which contained patterns of ions typical of sesquiterpene hydrocarbons (Joulain and Konig 1998); and their GC retention times on a nonpolar DB5-MS column, which fell between those of tetradecane and hexadecane. These compounds were tentatively identified as $(E)$ - $\beta$-farnesene, $\alpha$-curcumene, zingiberene, $\beta$-bisabolene, and the male specific compound $\beta$-sesquiphellandrene by mass spectral matches with database spectra. 
Table 1 Compounds identified in volatiles collected from sexually mature virgin adult $P$. guildinii

\begin{tabular}{lll}
\hline Compound & \multicolumn{2}{l}{ Amount $\left(\%\right.$, Mean \pm SE) Extract from Volatile Collections ${ }^{\mathrm{a}}$} \\
\cline { 2 - 3 } & Male $(N=4)$ & Female $(N=3)$ \\
\hline$(E)$-2-Hexenal & $13.0 \pm 5.3$ & $1.9 \pm 0.8$ \\
$\alpha$-Pinene & $20.6 \pm 6.3$ & $5.9 \pm 1.2$ \\
Ocimene & $0.6 \pm 0.3$ & $4.8 \pm 1.8$ \\
$(E)$-2-Hexenyl acetate & $1.4 \pm 0.7$ & $15.1 \pm 1.5$ \\
2-Ethylhexanol & $0.3 \pm 0.1$ & $3.6 \pm 2.2$ \\
Undecane & $2.6 \pm 0.5$ & $4.4 \pm 1.9$ \\
Nonanal & $0.7 \pm 0.4$ & $3.8 \pm 2.3$ \\
Dodecane & $2.2 \pm 1.5$ & $39.4 \pm 13.8$ \\
Indole & $0.3 \pm 0.2$ & $6.2 \pm 1.4$ \\
Tridecene & $0.7 \pm 0.1$ & $13.5 \pm 6.8$ \\
Tridecane & 100 & 100 \\
$(E)$ - $\beta$-farnesene & $29.9 \pm 11.9$ & $6.2 \pm 5.9$ \\
$\alpha$-Curcumene & $3.5 \pm 2.4$ & $0.10 \pm 0.05$ \\
Zingiberene & $3.3 \pm 3.1$ & $0.10 \pm 0.03$ \\
$\beta$-Bisabolene & $5.4 \pm 3.5$ & $0.20 \pm 0.01$ \\
$\beta$-Sesquiphellandrene & $94.3 \pm 46.3$ & $0 \pm 0$ \\
\hline
\end{tabular}

${ }^{a}$ The mean percentages of compounds relative to tridecane, individually produced by each sex, were obtained from volatiles collection from the first day of collection, where the insects were stressed by manipulation.

The identifications were confirmed by mass spectral and retention time matches with those of authentic standards. All these compounds were of insect origin because they were not present in control aerations of the green beans used as food during aerations (Borges et al. 2006).

Despite the care taken in loading stink bugs into aeration chambers, defensive compounds were still obtained in large quantities on the first day of extraction (Table 1 and Fig. 2). Thus, these extracts were not used for bioassays or for fractionation. Extracts obtained from the second day onward contained low levels of defensive compounds (Fig. 2), and thus were used for analyses and bioassays.

The sesquiterpenes were seen in all extracts $(N=150)$ collected from males, whereas these compounds were seen only in trace quantities in a small proportion (15 of 150) of female extracts (Table 1). $\beta$-Sesquiphellandrene was never seen in any of the 150 extracts from females that were analyzed. Furthermore, $\beta$-sesquiphellandrene was detected only in extracts from sexually mature males ( $>8 \mathrm{~d}$ old) (Fig. 2).

The absolute configuration of $\beta$-sesquiphellandrene was determined by two indirect routes because its enantiomers were not resolved on the Cyclodex B GC column. First, the insect-produced $\beta$-sesquiphellandrene was dehydrogenated with 3,4,5,6-tetrachloro-1,2benzoquinone to produce the aromatic analog, $\alpha$-curcumene, which was resolved on the chiral column, with the (7S)-enantiomer eluting first (McBrien et al. 2002). The $\alpha$ curcumene derived from $\beta$-sesquiphellandrene produced by male $P$. guildinii proved to be the $(7 R)$-enantiomer. However, because the dehydrogenation reaction gave a poor yield of $\alpha$-curcumene, a second method was developed to confirm the absolute configuration of the insect-produced $\beta$-sesquiphellandrene. Thus, standards of stereoisomerically pure $(7 S)$ - $\beta$ sesquiphellandrene isolated from ginger oil, synthetic (7R)- and (7S)-sesquiphellandrenes, and the insect-produced $\beta$-sesquiphellandrene were hydrogenated, producing a $\sim 2.5: 1$ mixture of diastereomers. On the Cyclodex B column, the more abundant, earlier-eluting 
Fig. 2 Typical gas chromatograms of volatiles collected from male (top) and female (bottom, inverted) $P$. guildinii when the insects are not stressed: $1=(E)$ 2-hexenal, 2 =ocimene isomer, 3=2-ethylhexanol, $4=$ undecane, $5=$ nonanal, $6=$ tridecene, $7=$ tridecane, $8=\alpha$-farnesene, $9=\beta$ sesquiphellandrene

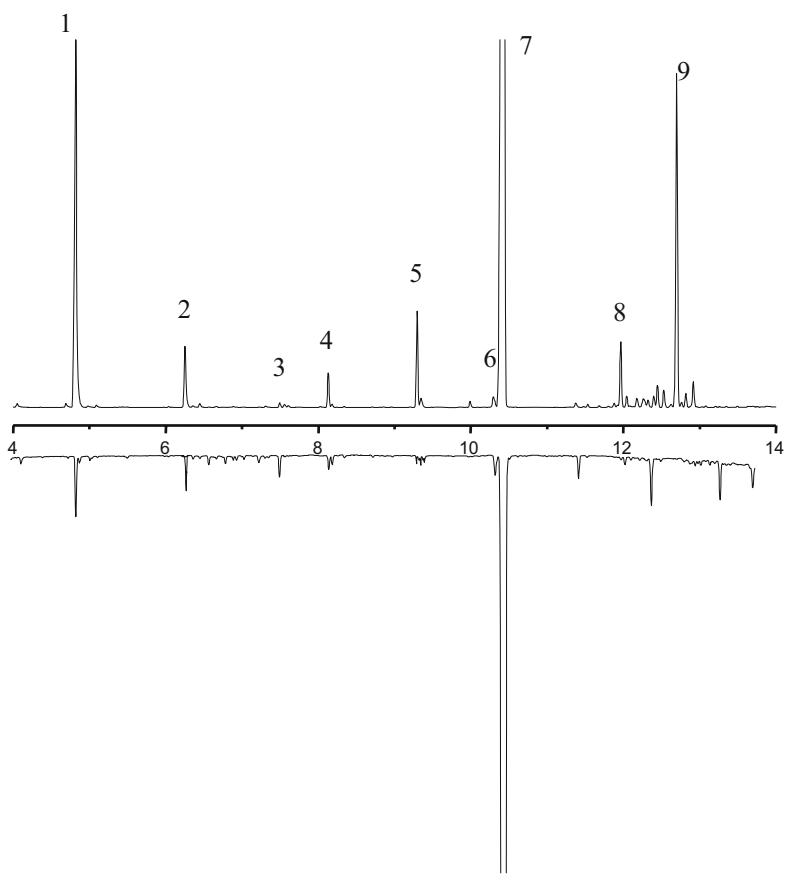

diastereomer was not resolved into its enantiomers. However, the minor diastereomer derived from each enantiomer was partially resolved [derivative from $(S)$-enantiomer, $133.13 \mathrm{~min}$; from $(R)$-enantiomer, $134.01 \mathrm{~min}]$. Coinjection of the reduced $\beta$-sesquiphellandrene collected from the insects with the reduced $(7 S)$ - $\beta$-sesquiphellandrene standard confirmed that the insect-produced material had the $(7 R)$-configuration.

Analysis of 10 extracts obtained from groups of 20 males aerated for $24 \mathrm{hr}$ determined that males released approximately $40 \mathrm{ng}$ of sesquiphellandrene per bug per day. There was no clear cycle of production, with males producing similar amounts during morning, afternoon, and evening (Fig. 3, repeated measures ANOVA, $F_{2,8}=0.21, P=0.81$ ).

Bioassays with Live Insects Female bugs were attracted to odors of live males $\left(\chi_{1}^{2}=10.76\right.$, $N=41, P=0.001$; Fig. 4) but not to odors of live females $\left(\chi_{1}^{2}=0.13, N=32, P=0.72\right.$; Fig. 4). Males were not attracted to odors of females $\left(\chi_{1}^{2}=0.28, N=14, P=0.59\right.$; Fig. 4$)$ or of males. In the latter case, most of the males tested (24/30) did not leave the release chamber of the olfactometer, and so statistical analysis was not performed for the six individuals that made a choice (four to control, two to treatment; Fig. 4). When female and male P. guildinii were tested with the crude extract of males, female bugs were attracted to the extract of male odor $\left(\chi_{1}^{2}=18.06, N=64, P<0.001\right.$; Fig. 5a), whereas males were not (Fig. 5a). These results indicated that males produced a sex-specific pheromone that attracted only females, and so all further bioassays were done only with females as test individuals, with the exception that males were also bioassayed to the synthetic standards to double-check that the compound was a sex pheromone rather than an aggregation pheromone.

Females' Responses to Crude Extract of Males at Different Times of the Day When females were tested during three different periods of the day (morning, afternoon, and evening), they responded to crude extracts of male-produced volatiles only in the evening. During the 


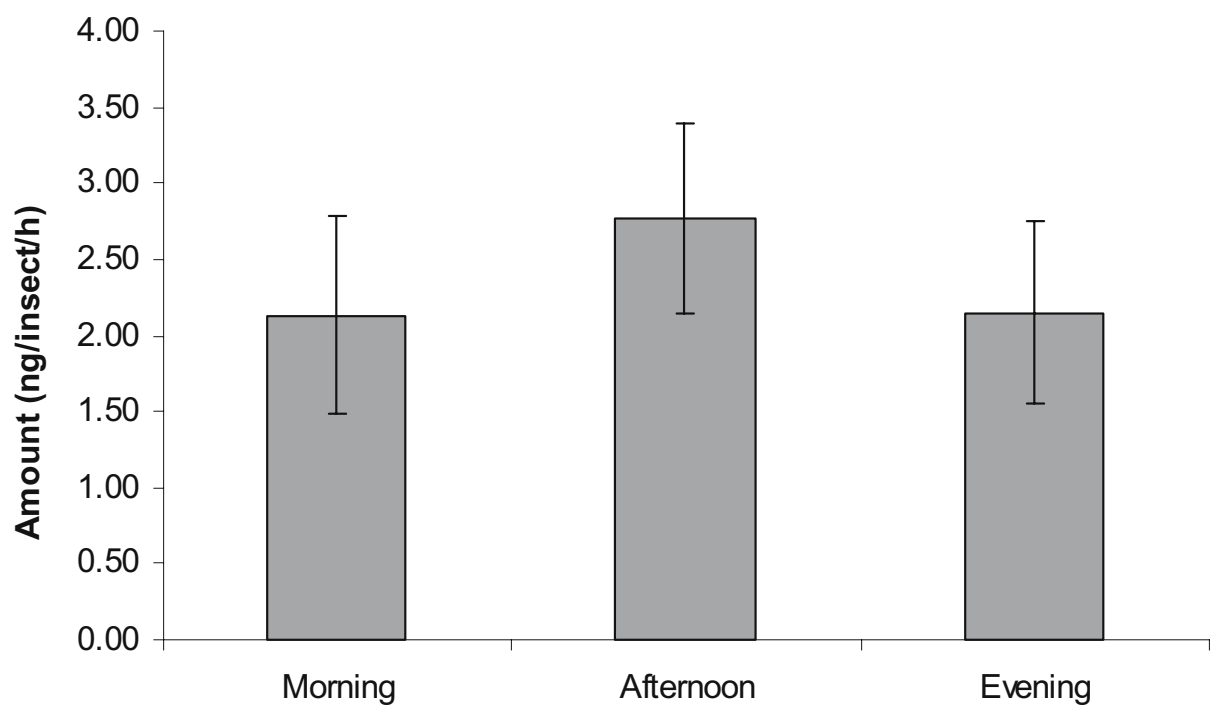

Fig. 3 Mean amount (ng insect ${ }^{-1} \mathrm{~h}^{-1} \pm \mathrm{SD}$ ) of pheromone released by male $P$. guildinii during different periods of the day. Collections lasted $5 \mathrm{hr}$ for each period. The amounts collected during the three periods were not significantly different (repeated measures ANOVA, $F_{2,8}=0.35, P=0.72$ )

evening, $70.3 \%$ of the females tested chose the treatment chamber $\left(\chi_{1}^{2}=25.83, N=53, P<0.001\right.$; Fig. $5 \mathrm{~b})$, whereas for the morning only $4.3 \%(1 / 23)$ of females tested left the release chamber of the olfactometer (Fig. 5b). In the afternoon, the test insects were more active $(69.5 \%$ moving out of the release chamber) but their responses were not oriented toward the treatment chamber

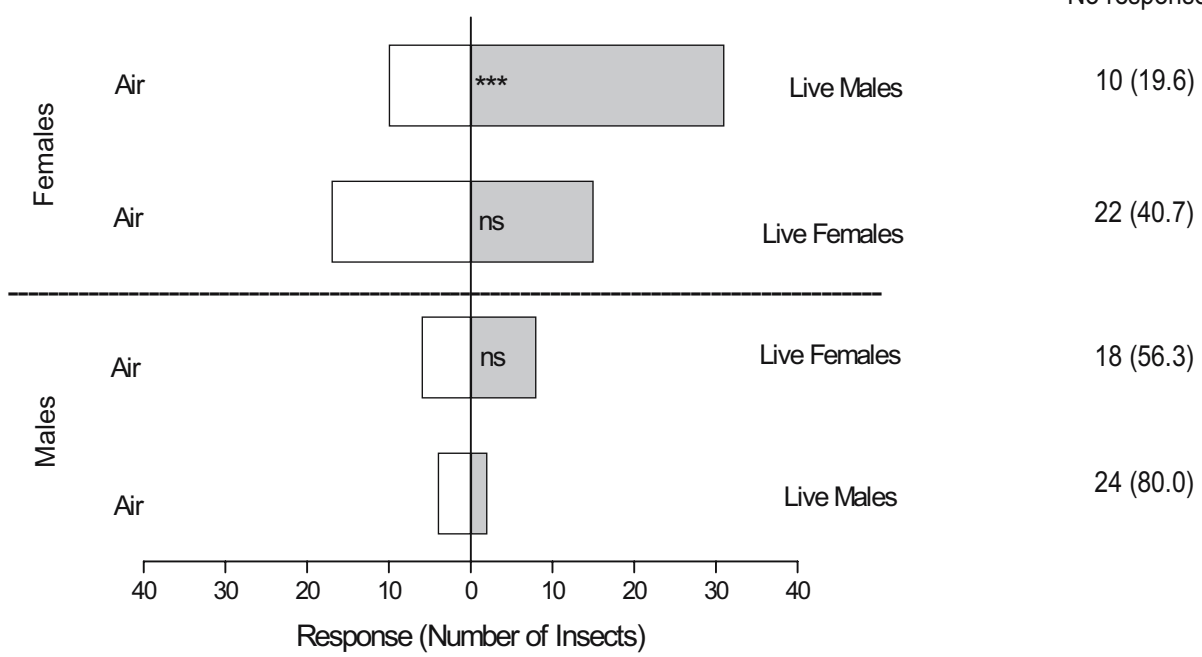

Fig. 4 Responses of male and female $P$. guildinii to odor of conspecifics of each sex vs. blank controls, in a two-choice olfactometer bioassay. Data were analyzed by chi-square tests (ns $P>0.05, * * * P<0.001)$. Data for males responding to odor of live males were not analyzed because of the low number of responders $(6 / 30$ insects tested). The numbers of nonresponders are to the right of the figure. Numbers in parentheses represent the percentage of nonresponders out of the total number of insects tested 
$\left(\chi_{1}^{2}=0.36, N=25, P=0.55\right.$; Fig. 5B). Thus, during this period the females did not appear to be responsive to male pheromone. In light of these results, all further bioassays were performed from 17:00 to 22:00 hours.

Bioassays with Fractions of Insect Extracts and Synthetic Standards When the two fractions of crude extract were bioassayed against the hexane control, female $P$. guildinii responded to fraction $\mathrm{A}$, which contained primarily $(7 R)-\beta$-sesquiphellandrene $\left(\chi_{1}^{2}=17.0\right.$, $N=68, P<0.001$; Fig. $5 \mathrm{c}$ ). Females did not respond to fraction $\mathrm{B}$, which contained primarily saturated hydrocarbons and several sesquiterpenes $\left(\chi_{1}^{2}=0.28, N=14, P=0.59\right.$; Fig. 5c). Females also preferred fraction $\mathrm{A}$ when it was evaluated directly vs. fraction $\mathrm{B}\left(\chi_{1}^{2}=7.54\right.$, $N=26, P=0.006$; Fig. 5c). There were no differences in the responses of female bugs to fraction $\mathrm{A}$ in bioassays against the crude extract $\left(\chi_{1}^{2}=0.05, N=21, P=0.83\right.$; Fig. 5c), indicating that fraction A was responsible for the attractiveness of the crude extracts. Furthermore, when fraction A, containing (7R)- $\beta$-sesquiphellandrene, was tested against an equal amount of the pure (7S)-enantiomer isolated from ginger oil, fraction A was more attractive $\left(\chi_{1}^{2}=10.67, N=24, P=0.001\right.$; Fig. $\left.5 \mathrm{c}\right)$. In addition, when synthetic $(7 R)$ - and $(7 S)$ - $\beta$-sesquiphellandrenes were tested individually against hexane controls, the $(7 R)$ $\beta$-sesquiphellandrene was attractive to females, whereas the $(7 S)$-enantiomer was not $\left(\chi_{1}^{2}=\right.$ 4.83, $N=35, P=0.03$; Fig. 6a). In contrast, male bugs showed no response to either enantiomer of the synthetic sesquiphellandrene against hexane controls (Fig. 6a). When a 1:1 mixture of synthetic $(7 R)$ - and $(7 S)$ - $\beta$-sesquiphellandrenes was tested against each enantiomer individually, only the $(7 R)-\beta$-sesquiphellandrene was as attractive as the $1: 1$ mixture $\left(\chi_{1}^{2}=\right.$ $0.33, N=48, P=0.56$; Fig. $6 \mathrm{~b})$. However, when synthetic $(7 R)-\beta$-sesquiphellandrene was tested vs. synthetic (7S)- $\beta$-sesquiphellandrene, females responded equally to both treatments $\left(\chi_{1}^{2}=0.09, N=46, P=0.77\right.$; Fig. $\left.6 \mathrm{~b}\right)$. There was no difference in the response of female bugs to fraction $\mathrm{A}$ in bioassays vs. synthetic (7R)- $\beta$-sesquiphellandrene (Fig. $6 \mathrm{~b}$ ), indicating that fraction A, containing male-produced (7R)- $\beta$-sesquiphellandrene, was as attractive as synthetic (7R)- $\beta$-sesquiphellandrene to females. Furthermore, when fraction A was tested against the synthetic $(7 S)$-enantiomer, fraction $\mathrm{A}$ was more attractive than the $(7 S)$-enantiomer $\left(\chi_{1}^{2}=10.67, N=24, P=0.001\right.$; Fig. $\left.6 b\right)$.

\section{Discussion}

Daily collections of volatiles $(N=150)$ from groups of 20 P. guildinii males, starting $5 \mathrm{~d}$ after the final molt, and continuing for 15 consecutive days, showed that young males did not produce detectable levels of male-specific compounds. Male-specific compounds were first detected in extracts of males $8 \mathrm{~d}$ after the molt to adulthood. Although the pheromone was collected from male $P$. guildinii throughout the day, female bugs were only attracted to volatiles from males during the evening, suggesting that females are only responsive during this period. These results are analogous to previous reports of mating behavior of the stink bugs Euschistus heros and Thyanta perditor in which the strongest responses to pheromone occurred during late afternoon to early evening (Borges et al. 1998a,b; Moraes et al. 2005b).

Most of the putative defensive compounds identified in the headspace volatiles of $P$. guildinii adults consisted of compounds that have been reported previously from other neotropical stink bug species (Borges and Aldrich 1992; Moraes et al. 2005b; Borges et al. 2006). However, we found that, in addition to $\beta$-sesquiphellandrene, males consistently released several sesquiterpenes. In contrast, sesquiterpenes were only seen in trace amounts 


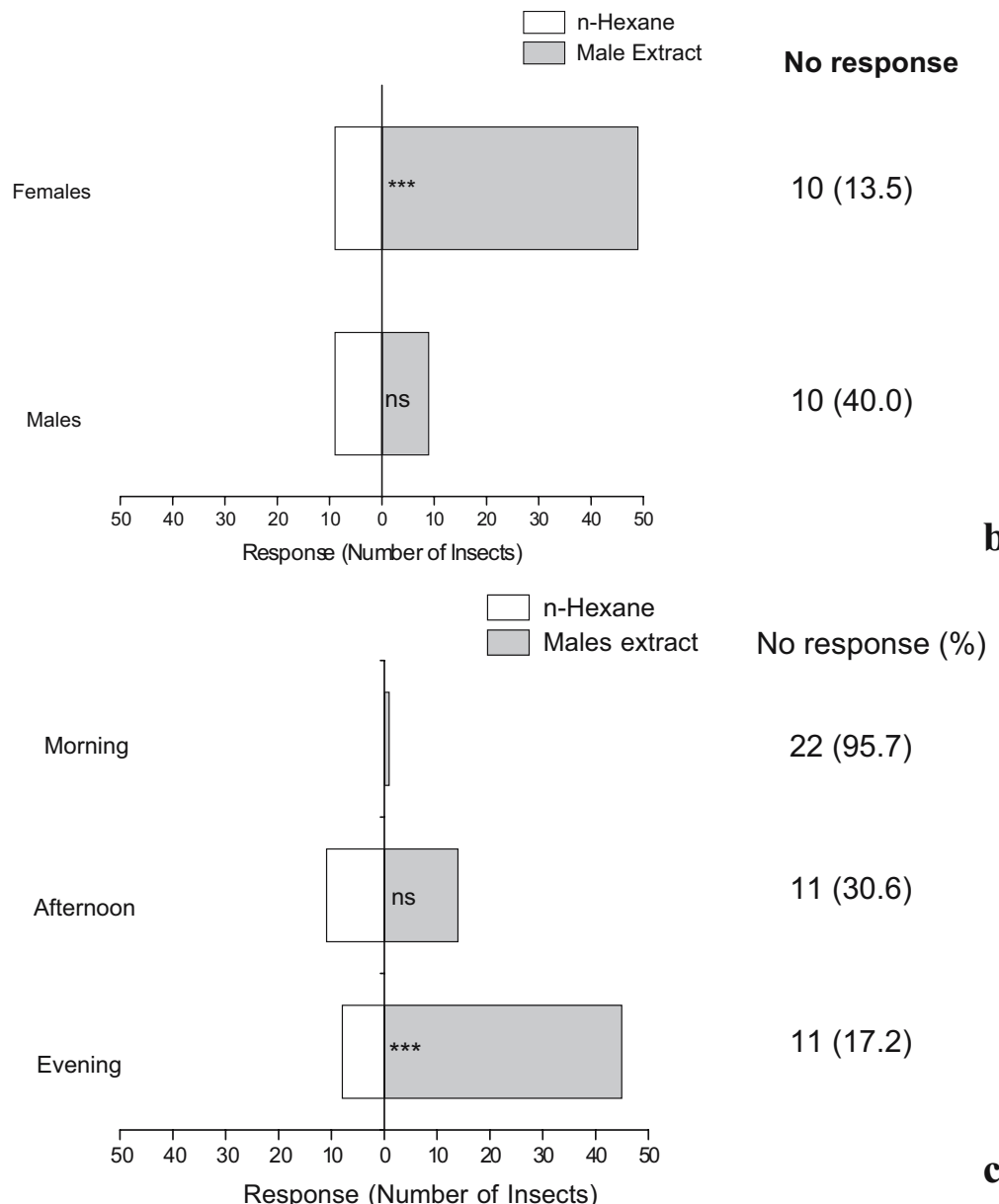

No response

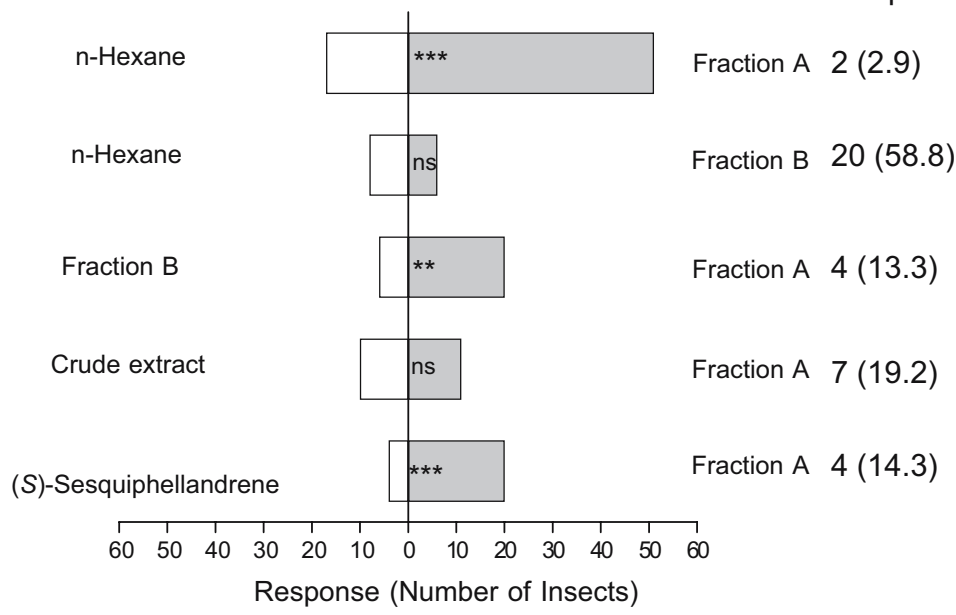


in a small percentage of extracts from females. The function of these sesquiterpenes, and why their production is strongly male-biased, is unknown. They did not appear to have a role in sexual attraction because $(7 R)$ - $\beta$-sesquiphellandrene alone was as attractive as crude extracts. They also do not appear to be artifacts from the green beans provided as food because they were not detected in extracts of volatiles from the green beans.

The first study of pheromones within the genus Piezodorus was carried out by Leal et al. (1998) with $P$. hybneri. The pheromone blend of $P$. hybneri, which attracted both sexes in laboratory bioassays, consisted of $\beta$-sesquiphellandrene (absolute configuration not determined), $(R)$-15-hexadecanolide, and methyl (Z)-8-hexadecenoate (ratio 10:4:1). The responses of females to subsets of the blend or individual compounds were not investigated (Leal et al. 1998). Thus, it remains unclear whether the full three-component blend is required to attract females, or whether the attraction observed in bioassays was actually because of only one or two components of the blend.

In contrast, the pheromone of congeneric $P$. guildinii appears to consist of a single component, $(7 R)-\beta$-sesquiphellandrene, which attracts only females. Furthermore, examination of the percentage of nonresponders in bioassays (Figs. 4, 5, and 6b) showed that in all bioassays in which the natural compound $(7 R)$ - $\beta$-sesquiphellandrene was present (live males, airborne extracts, fraction A from airborne extracts), the percentages of females that did not respond were lower than those in bioassays in which the treatments did not include this compound (Figs. 4, 5, and 6b). It is also interesting to note that $\beta$-sesquiphellandrene produced by $P$. guildinii has the opposite configuration to the $(7 S)-\beta$-sesquiphellandrene produced as a pheromone component by two other stink bug species, Thyanta pallidovirens and Thyanta custator accerra (McBrien et al. 2002).

Although the results from most of our bioassays indicated that the insect-produced $(7 R)$ enantiomer of $\beta$-sesquiphellandrene was the active antipode, in one test similar numbers of bugs responded to both synthetic enantiomers (Fig. 6b). In addition, in some bioassays testing synthetic compounds, there were more nonresponding test bugs than in bioassays with the insect-produced material (Fig. 6). The cause of these anomalies is not clear. It may have been because of the fact that the synthetic compounds were both mixtures of epimers, rather then pure compounds, thereby distorting the olfactory signal. It was not because of large amounts of the $(7 R)$-enantiomer in the synthetic $(7 S)$-enantiomer because the latter compound was highly enantiomerically pure $(>98 \%$ ee).

In a preliminary report, Borges et al. (1999) reported the identification of methyl 2,6,10trimethyltridecanoate and methyl 2,6,10-trimethlyldodecanoate in extracts of field-collected P. guildinii. They (Borges et al. 1999) reported that these compounds appeared to be attractive to $P$. guildinii, based on catches in traps, that were deployed to catch E. heros. For $E$. heros, these two esters have been identified in volatiles collected from male bugs, and the biological activities of the esters verified in both laboratory bioassays and field tests (Borges and Aldrich 1994; Borges et al. 1998a,b; Costa et al. 2000; Zhang et al. 2003). In contrast, these compounds were not found in any of the samples analyzed in the current

Fig. 5 Response of $P$. guildinii adults in two-choice olfactometer bioassays. a Responses of males and females to odor extracts of males vs. solvent controls. b Responses of females to extracts of males vs. solvent controls during different periods of the day. Results from the morning period were not analyzed because only 1 of 23 insects responded. c Responses of females to crude extracts of males, fractions A [containing (7R)$\beta$-sesquiphellandrene] and B (containing saturated hydrocarbons and several other sesquiterpenes) of crude extracts of males, and (7S)- $\beta$-sesquiphellandrene isolated from ginger oil vs. solvent controls. Data were analyzed with chi-square tests (ns $P>0.05$, ** $P<0.01, * * * P<0.001$ ). The numbers of nonresponders are to the right of the figure. Numbers in parentheses represent the percentage of nonresponders out of the total number of insects tested 

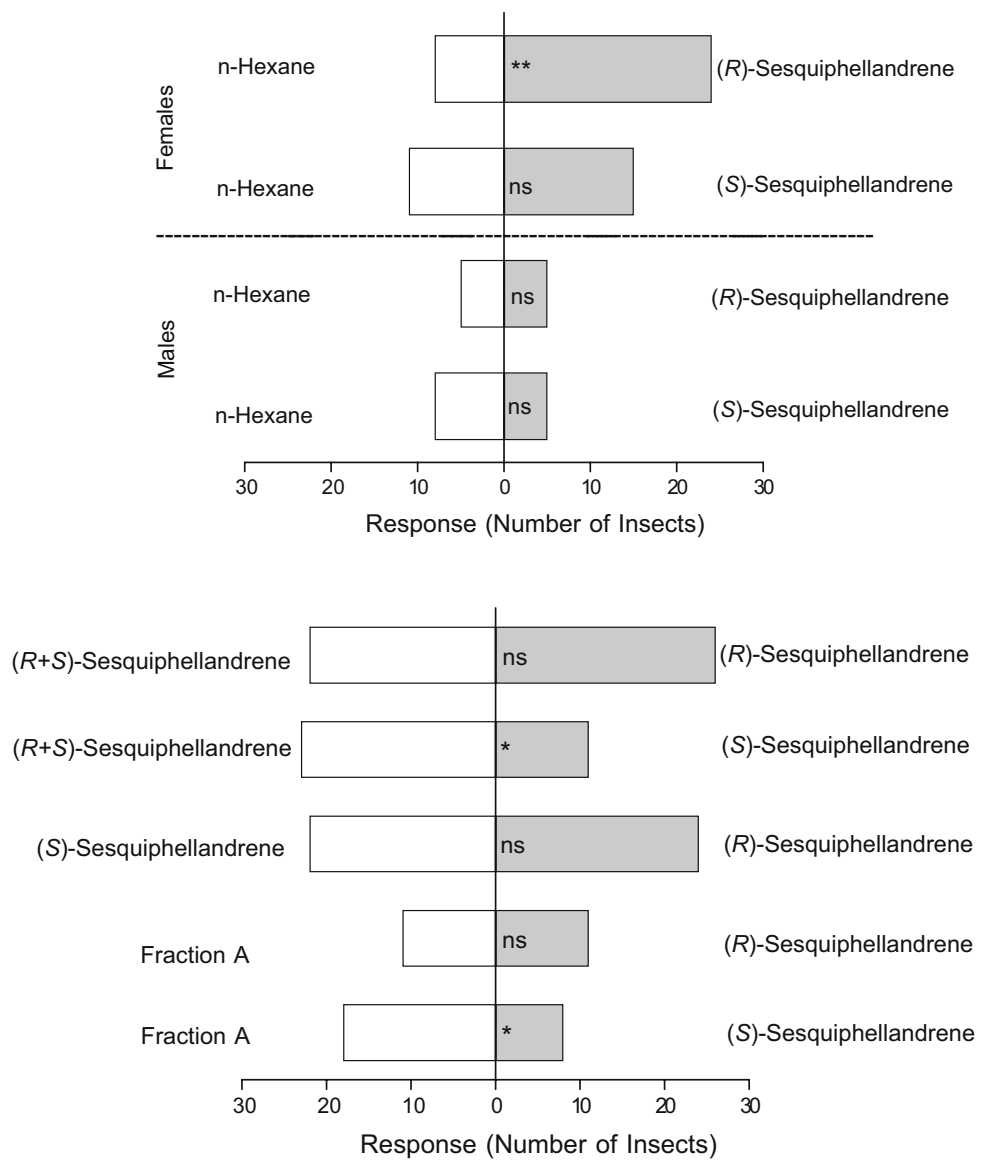

No response (\%)

$20(36.4)$

b

No response (\%)

$24(33.3)$

$31(47.7)$

$34(42.5)$

$17(43.6)$

$14(14.3)$

Fig. 6 Responses of $P$. guildinii in two-choice olfactometer bioassays. a Response of males and females to synthetic (7R)- or (7S)- $\beta$-sesquiphellandrene vs. solvent controls. b Response of females to synthetic (7R)- or (7S)- $\beta$-sesquiphellandrene vs. each other, vs. a 1:1 mixture of both compounds, and vs. fraction A [containing insect-produced (7R)- $\beta$-sesquiphellandrene]. Data were analyzed with chi-square tests (ns $P>$ $0.05, * * P<0.01, * * * P<0.001)$. The numbers of nonresponders are to the right of the figure. Numbers in parentheses represent the percentage of nonresponders out of the total number of insects tested

study. It should be pointed out that the previous identifications were based only on GC retention time matches to the two esters, which on more detailed investigation by gas chromatography-mass spectrometry appear to be coincidental. The basis for the attraction of P. guildinii to the esters (Borges et al. 1999; Aldrich et al. 1993) is unclear. However, a similar phenomenon has been observed for other stink bug species in which bugs appear to be attracted to the pheromone components of other bug species. For example, the Asian species Halyomorpha halys (Stål) is attracted to ester pheromone components of Plautia stali (Scott) and of Thyanta spp., although the esters have not been found in Halyomorpha bugs (J. R. Aldrich, personal communication). In addition, Endo et al. (2007) reported cross attraction of P. hybneri to the aggregation pheromone of Riptortus clavatus (Thunberg). 
The role of $(7 R)-\beta$-sesquiphellandrene as a mediator of $P$. guildinii reproductive behavior under field conditions still remains to be determined. It is likely that the pheromone may act in concert with the substrate-borne vibrational signals that this insect uses for communication over shorter ranges (Moraes et al. 2005a). It may be possible to develop pheromone-based monitoring systems for this insect, particularly if vibrational signals could also be incorporated into the trap design. Such monitoring systems, when used in conjunction with strategies such as trap cropping, may provide an integrated pest management solution for control of this important pest.

Acknowledgements We thank Hélio Santos, Diva Tiburcio, and Camila Raquel for helping with field collection and laboratory rearing of the insects used in this study. This work received financial support from the Brazilian Council for Scientific and Technological Development (CNPq), Distrito Federal Research Foundation (FAPDF), and EMBRAPA. This research was also supported by the International Foundation for Science, Stockholm, Sweden, through a grant to Maria Carolina Blassioli Moraes.

\section{References}

Aldrich, J. R., Numata, H., Borges, M., Bin, F., Waite, G. K., and Lusby, W. R. 1993. Artifacts and pheromone blends from Nezara spp. and other stink bugs (Heteroptera: Pentatomidae). Z. Naturforsch. 48C:73-79.

Borges, M. and Aldrich, J. R. 1992. Instar-specific defensive secretions of stink bugs (Heteroptera: Pentatomidae). Experientia 48:893-896.

Borges, M. and BlDRICH, J. R. 1994. Attractant pheromone for nearctic stink bug, Euschistus obscurus (Heteroptera: Pentatomidae): insight into a neotropical relative. J. Chem. Ecol. 20:1095-1102.

Borges, M., Mori, K., Costa, M. L. M., and SujI, E. R. 1998a. Behavioural evidence of methyl-2,6,10trimethyltridecanoate as a sex pheromone of Euschistus heros (Heteroptera: Pentatomidae). J. Appl. Entomol. 122:335-338.

Borges, M., Schmidt, F. G. V., SuJi, E. R., Medeiros, M. A., Mori, K., Zarbin, P. H. G., and Ferreira, T. B. 1998b. Field responses of stink bugs to the natural and synthetic pheromone of the neotropical brown stink bug, Euschistus heros (Heteroptera: Pentatomidae). Physiol. Entomol. 23:202-207.

Borges, M., Zarbin, P. H. G, Ferreira, J. T. B., and Costa, M. L. M. 1999. Pheromone sharing: blends based on the same compounds for Euschistus heros (F.) and Piezodorus guildinii (W.) (Heteroptera: Pentatomidae). J. Chem. Ecol. 25:629-634.

Borges, M., Birkett, M., Aldrich, J., A., Oliver, J. E., Chiba, M., Murata, Y., Laumann, R. A., Barrigossi, J. A., Pickett, J. A., and Moraes, M. C. B. 2006. Sex attractant pheromone from the rice stalk stink bug, Tibraca limbativentris Stal. J. Chem. Ecol. 32:2749-2761.

Chavan, S. P., Dhondge, V. D., and Patil, S. S. 1997. Enantiospecific total synthesis of (+)-laevigatin. Tetrahedron: Asymmetry 8:2517-2518.

CorrêA-FERreIRA, B. S. and Moscardi, F. 1996. Biological control of soybean stink bugs by inoculative releases of Trissolcus basalis. Entomol. Exp. Appl. 79:1-7.

Costa, M. L. M., Borges, M., and Vilela, E. F. 2000. Effect of stereoisomers of the main component of the sex pheromone of Euschistus heros (F.) (Heteroptera: Pentatomidae) in the attractiveness of female. An. Soc. Entomol. Bras. 29:413-422.

ENDO, N., WADA, T., NishiBA, Y., and SASAKI, R. 2007. Interspecific pheromone cross-attraction among soybean bugs (Heteroptera): does Piezodorus hybneri (Pentatomidae) utilize the pheromone of Riptortus clavatus (Alydidae) as a kairomone? J. Chem. Ecol. 32(7):1605-1612.

JACKMAN, L. M. 1960. Hydrogenation-dehydrogenation reactions, pp. 329-364, in R. Raphael, E. C. Taylor, and H. Wynberg (eds.). Advances in Organic Chemistry. Methods and Results, vol. II. Interscience Publishers, New York.

Joulain, D. and Konig, W. A. 1998. The Atlas of Spectral Data of Sesquiterpene Hydrocarbons. E.B.Verlag, Hamburg.

Leal, S. W., Kuwahara, X. S., Higuchi, H., Marino, C. E. B., Ono, M., and Meinwald, J. 1998. Malereleased sex pheromone of the stink bug Piezodorus hybneri. J. Chem. Ecol. 24:1817-1829.

Mcbrien, H. L., Millar, J. G., Rice, R. E., Mcelfresh, J. S., Cullen, E., and Zalom, F. G. 2002. Sex attractant pheromone of the red-shouldered stink bug Thyanta pallidovirens: a pheromone blend with multiple redundant components. J. Chem. Ecol. 28:1797-1818. 
Millar, J. G. 1998. Rapid and simple isolation of zingiberene from essential oils. J. Nat. Prod. 61:1025-1026.

Moraes, M. C. B., Laumann, R. A., CoKL, A., and Borges, M. 2005a. Vibratory signals of four Neotropical stink bug species. Physiol. Entomol. 30:175-188.

Moraes, M. C. B., Millar, J. G., Laumann, A. R., Sujit, E. R., Pires, C. S. S., and Borges, M. 2005 b. Sex attractant pheromone from the neotropical red-shouldered stink bug, Thyanta perditor (F.). J. Chem. Ecol. 31:1415-1427.

PANIZZI, A. R. and Rossi, C. E. 1991. The role of Acanthospermum hispidum in the phenology of Euschistus heros and Nezara viridula. Entomol. Exp. Appl. 59:67-74.

ShePARD, M., LAWN, R. J., and SchneIDER, M. A. 1983. Insects on Grain Legumes in Northern Australia. A Survey of Potential Pests and Their Enemies. Univ. Queensland Press, St. Lucia, Australia.

Singh, O. P., SingH, K. J., and ThaKUR, R. D. 1989. Studies on the bionomics and chemical control of stink bug, Piezodorus rubrofasciatus Fabricius, a new pest of soybean in Madhya Pradesh. Ind. J. Plant Prot. 17:81-93.

Zarbin, H. G. P., Borges, M., Santos A. A., Oliveira, A. R. M., Simonelli, F., and Marques, F. A. 2000. Alarm pheromone system of stink bug Piezodorus guildinii (Heteroptera: Pentatomidae). J. Braz. Chem. Soc. 11:424-428.

Zhang, A., Borges, M., Aldrich, J. R., and CAmp, M. J. 2003. Stimulatory bug volatiles for the neotropical brown stink bug, Euschistus heros (F.) (Heteroptera: Pentatomidae). Neotrop. Entomol. $32: 713-717$. 